\title{
Mitogenic factor (MF) is the major DNase of serotype M89 Streptococcus pyogenes
}

\author{
Shiranee Sriskandan, Meera Unnikrishnan, Thomas Krausz \\ and Jonathan Cohen
}

Author for correspondence: Shiranee Sriskandan. Tel: +44208383 3135. Fax: +44 2083833394. e-mails.sriskandan@ic.ac.uk

Department of Infectious Diseases, Imperial College School of Medicine, London W12 0NN, UK

\begin{abstract}
To investigate the role of mitogenic factor (MF) in streptococcal pathogenesis, the structural gene $(\mathrm{mf})$ encoding this protein was disrupted in a clinical isolate of Streptococcus pyogenes H293, to yield the isogenic mutant $\mathrm{H} 363$. Growth in enriched broth and on blood agar was unaffected by disruption of mf. Cell-free broth supernatants from H293 and H363 demonstrated identical promitogenic activities when co-incubated with human peripheral blood mononuclear cells, even when diluted 100000 -fold, showing that MF is not a major streptococcal mitogen compared with other secreted superantigens. Disruption of $\boldsymbol{m f}$ resulted in complete loss of DNase B production and detectable DNase activity in $\mathrm{H} 363$ compared with the parent strain, confirming that the single gene $m f$, which is present in all group A streptococcal $M$ serotypes studied, encodes DNase B. Despite loss of DNase activity, the virulence of $S$. pyogenes in a mouse model of necrotizing fasciitis and myositis was unaffected.
\end{abstract}

Keywords: superantigen, nuclease, mouse model, necrotizing fasciitis

\section{INTRODUCTION}

The virulence of Streptococcus pyogenes is attributed both to an inherent resistance to opsonophagocytosis in non-immune whole blood and to a remarkable repertoire of secreted enzymes and toxins. Over the last decade, considerable attention has focused on the role of superantigens in streptococcal disease pathogenesis and toxic shock, in particular the ability of superantigens to cause clonal $\mathrm{T}$ cell proliferation and cytokine release (Herman et al., 1991). Superantigens such as streptococcal pyrogenic exotoxins (SPEs) A and C are expressed only by phage-carrying strains, whereas the newly recognized structurally related streptococcal mitogenic exotoxin Z (SMEZ) and SPEG appear to be chromosomally encoded (Proft et al., 1999). Mitogenic factor (MF, also termed SPEF) is a chromosomally encoded streptococcal toxin with apparently similar mitogenic properties to the above superantigens, though with quite distinct structure (Iwasaki et al., 1993; Toyosaki et al., 1996). It is well recognized that streptococci that lack the phage-encoded superantigens SPEA and SPEC can cause severe invasive disease with toxic shock (Davies et al., 1996; Zurawski et al., 1998); we therefore concluded

Abbreviations: IL-6, interleukin-6; MF, mitogenic factor; PBMC, peripheral blood mononuclear cells. that chromosomally encoded virulence factors must account for the lethality of such strains.

Recombinant MF is reported to have nuclease activity and separate studies have shown that MF and the streptococcal nuclease DNase B share identical immunologic epitopes (Eriksson et al., 1999; Iwasaki et al., 1997), suggesting that the two proteins are equivalent. Antibodies to both DNase B and MF have been detected in patients recovering from streptococcal sepsis, showing that these proteins are synthesized by pathogenic streptococci during infection (Gerber et al., 1980; Norrby-Teglund et al., 1994a). Patients with toxic shock, however, have lower neutralizing antibody titres against MF than those with uncomplicated streptococcal disease, suggesting a pathogenic role for this protein (Norrby-Teglund et al., 1994a). In this study, we set out to characterize the pathogenic role of MF in a $S$. pyogenes isolate which lacks the known phage-encoded superantigens, by disruption of the chromosomal copy of the gene $m f$.

\section{METHODS}

Bacteria, media and antibiotics. S. pyogenes strain $\mathrm{H} 293$ (M89, PT4245 typed by the Central Public Health Laboratory, Colindale, London) was isolated from thigh muscle tissue of a patient with necrotizing fasciitis and sepsis syndrome. Toxin 
typing by PCR confirmed that the isolate was negative for speA, speC, speH and ssa, but positive for $m f$, spe $G$ and smeZ. PCR primers for $s p e C$ and ssa were designed from the published gene sequences. PCR primers for speA, $m f$, smeZ, spe $G$ and speH have been previously described by Proft et al. (1999) and Unnikrishnan et al. (1999). Streptococci were grown in Todd-Hewitt (TH) broth with $0.2 \%$ yeast extract (THY; Oxoid) or on solid nutrient agar with $5 \%$ horse blood. In some cases, streptococci were grown in TH broth alone. Escherichia coli (Novablue cells; Novagen) were grown in Luria-Bertani (LB) broth or on solid LB agar. Kanamycin was used to select for transformants $\left(400 \mu \mathrm{g} \mathrm{ml}^{-1}\right.$ for streptococci and $25 \mu \mathrm{g} \mathrm{ml}^{-1}$ for E. coli).

Plasmids and transformations. A HindIII 346 bp internal fragment of the $m f$ gene corresponding to nucleotides 943-1289 of the published sequence (Iwasaki et al., 1993) was amplified from strain $\mathrm{H} 305$ using primers MF1, GCAAGCTTCAAACACAGGTCTCA (forward), and MF2a, CGAAGCTTCCGACATAAGACAGACC (reverse). The HindIII-cut product was cloned into the suicide vector pUCMUT1, a derivative of pUC19 which has the aphA-3 kanamycin resistance gene flanked by two multi-cloning sites and no ampicillin resistance gene, to generate pUCMUT-mf (Sriskandan et al., 1999). Plasmid DNA was then extracted from E. coli and used to transform strain H293 by electroporation as previously described by Caparon \& Scott (1991) and Sriskandan et al. (1999), prior to kanamycin resistance selection. Transformation efficiency was standardized using a replicative control plasmid, pDL413, as previously described (Sriskandan et al., 1999).

Southern hybridization and PCR. Targeted insertional disruption of $m f$ caused by a single homologous recombination event between plasmid and chromosomal copies of $m f$ was confirmed initially by PCR, using primer pairs annealing to streptococcal chromosomal DNA upstream of the structural $m f$ gene (MFp, CTAGGTGACCACACAGCACC) and to the $3^{\prime}$ region of $m f$ distal to the intended region of homologous recombination (MF2, GCGAATTCGGTATAGCGCATGCCGCC).

For Southern hybridization, parent strain and transformant genomic DNAs were digested with NdeI, HindIII and SspI, electrophoresed, then transferred to nylon, hybridized with the 346 bp digoxigenin-labelled $m f$ fragment (amplified from H305 DNA using primers MF1 and MF2a) and developed with the DIG Detection kit (Boehringer Mannheim), prior to reprobing with $a p h A-3$ as previously described (Sriskandan et al., 1999).

\section{In vitro comparison of $\mathrm{H} 293$ and MF-negative mutant H363}

Growth analysis of mutants. Growth of parent and mutant strains was compared by inoculating separate $100 \mathrm{ml}$ volumes of THY broth with overnight streptococcal culture $(5 \mathrm{ml})$ and monitoring $\mathrm{OD}_{600}$ over $8 \mathrm{~h}$, until stationary phase was reached. Cultures were diluted and plated onto blood agar to relate measured $\mathrm{OD}_{600}$ to bacterial viable counts.

Promitogenic activity of $\mathrm{H} 293$ and H363. Human peripheral blood mononuclear cells (PBMC) from three normal volunteers were prepared by Ficoll density-gradient centrifugation (Pharmacia). Human PBMC were resuspended in tissue culture medium (RPMI 1640 with $10 \%$ FCS, $50 \mu \mathrm{g}$ penicillin $\mathrm{ml}^{-1}$, $50 \mu \mathrm{g}$ streptomycin $\mathrm{ml}^{-1}$ and $2 \mathrm{mM}$ glutamine) at $1 \times 10^{6}$ cells $\mathrm{ml}^{-1}$, and $198 \mu \mathrm{l}$ suspension plated per well in 96-well plates $\left(2 \times 10^{5}\right.$ cells per well). H293 and the transformant strain $\mathrm{H} 363$ were incubated at $37^{\circ} \mathrm{C}$ overnight in tissue culture medium without antibiotics. Both strains grew equally well based upon $\mathrm{OD}_{600}$ and plating onto blood agar. Cell-free supernatants were filter-sterilized, subjected to 10 -fold serial dilution and then co-incubated in $22 \mu \mathrm{l}$ volumes with PBMC for $96 \mathrm{~h}$. Final supernatant concentrations were therefore $1: 10,1: 100,1: 1000,1: 10000$ and $1: 100000$. Cell proliferation was measured by uptake of tritiated thymidine $[1 \mu \mathrm{Ci}(37 \mathrm{kBq})$ per well in a volume of $20 \mu \mathrm{l}]$ added $16 \mathrm{~h}$ prior to cell harvesting (Amersham Pharmacia Biotech).

DNase activity of $\mathrm{H} 293$ and H363. Cell-free supernatants $(5 \mu \mathrm{l})$ from overnight antibiotic-free $\mathrm{TH}$ cultures containing $\mathrm{H} 293$ or H363 were inoculated into wells cut into DNase agar containing methyl green indicator dye (Oxoid). Aliquots $(5 \mu \mathrm{l})$ of stationary-phase broth from $\mathrm{H} 293$ or H363 grown without antibiotic were streaked onto similar plates. Plates were then incubated for $5 \mathrm{~h}$ (in the case of supernatants) or $24 \mathrm{~h}$ at $37^{\circ} \mathrm{C}$ and inspected for evidence of DNA hydrolysis.

Northern analysis of $\mathrm{H} 293$ and H363. RNA was prepared from THY broth cultures of $\mathrm{H} 293$ and H363, sampled at three different growth phases as previously described (Unnikrishnan et al., 1999). RNA samples were run on agarose gel, blotted onto nylon, then hybridized with the $346 \mathrm{bp}$ digoxigeninlabelled $m f$ probe, prior to development using the ECL system (Amersham) as before.

Western analysis of supernatants from $\mathrm{H} 293$ and H363. For Western blotting, supernatants from overnight antibiotic-free $\mathrm{TH}$ broth containing $\mathrm{H} 293$ or $\mathrm{H} 363$ or $\mathrm{TH}$ alone were filtersterilized. Streptococcal cultures demonstrated equivalent growth, based upon $\mathrm{OD}_{600}$ and plating $\left(\mathrm{H} 293,1.4 \times 10^{8}\right.$ c.f.u. $\mathrm{ml}^{-1} ; \mathrm{H} 363,1 \cdot 1 \times 10^{8}$ c.f.u. $\mathrm{ml}^{-1}$ ). Ten microlitres of each supernatant were subjected to SDS-PAGE under reducing conditions. The proteins were electroblotted onto nitrocellulose, which was then incubated successively with rabbit polyclonal anti-DNase B (1:10000 dilution, a kind gift of Dr Dieter Gerlach, Jena, Germany), biotinylated goat anti-rabbit IgG, streptavidin-conjugated horseradish peroxidase, and developed (ECL system; Amersham). Streptococcal DNase B enzyme reagent $(5 \mu \mathrm{l}$ of a $10 \times$ solution; Wampole Laboratories) was run as a positive control.

\section{Comparison of $\mathrm{H} 293$ and $\mathrm{H} 363$ in vivo during sepsis}

Bacterial preparation. H293 and H363 were grown overnight in $100 \mathrm{ml}$ THY without antibiotic. Strains were washed and resuspended in up to $5 \mathrm{ml}$ sterile saline, to give similar densities. For intramuscular infection, bacterial suspensions were adjusted to $2 \times 10^{9}$ c.f.u. $\mathrm{ml}^{-1}$ (approx.). Aliquots of each inoculum were diluted and plated out on blood agar to verify doses given.

Survival. Male outbred CD1 mice weighing 20-25 g (Charles River) were used in all experiments. Animals received food and water ad libitum. Two groups of 15 mice from the same batch (i.e. identical age and weight) received $\sim 10^{8}$ c.f.u. $\mathrm{H} 293$ or H363 streptococcal suspension per mouse intramuscularly into the right thigh, as previously described (Sriskandan $e t$ al., 1996). Mice were monitored over a $7 \mathrm{~d}$ period and those reaching the pre-determined end point were humanely killed. At $7 \mathrm{~d}$, all surviving mice were killed; serum from cardiac puncture was frozen at $-20{ }^{\circ} \mathrm{C}$. The survival experiment was repeated once. All animal procedures were conducted within local and Home Office guidelines.

Bacteriology, histopathology and cytokine analysis. Three groups of five mice were infected with either H293 or H363 as above, or injected with saline and at $48 \mathrm{~h}$ after infection, mice were killed. Cardiac puncture was performed and tissues (right thigh, liver, spleen, kidney) were formalin-fixed, 


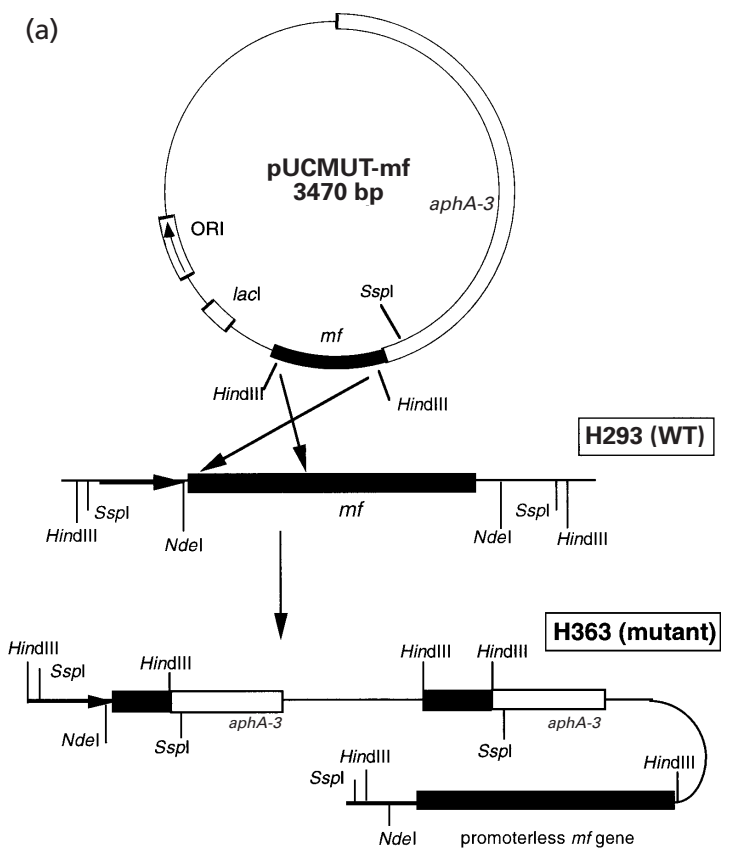

(b)
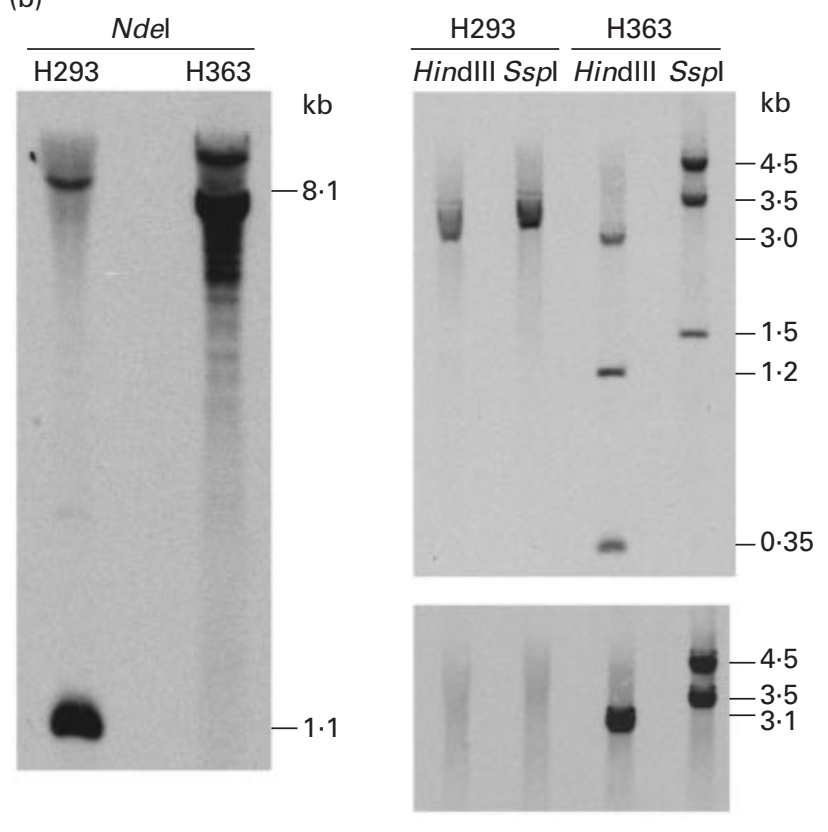

Fig. 1. (a). Mutation strategy using circular plasmid pUCMUT$\mathrm{mf}$ to transform wild-type (WT) S. pyogenes strain $\mathrm{H} 293$ chromosome, shown linearized. Black arrow on H293 DNA represents promoter for $m f$ gene and black bar represents $m f$ structural gene. Short black bar on pUCMUT-mf represents internal cloned segment of $m f$ gene, and adjacent white bar represents the $1.5 \mathrm{~kb}$ aphA-3 kanamycin resistance gene. A single cross-over event is shown between pUCMUT-mf and $\mathrm{H} 293$ chromosome, resulting in insertional mutation of the $\mathrm{mf}$ gene, shown in mutant H363. Note that insertion and duplication of plasmid pUCMUT-mf has occurred, separating native chromosomal $\mathrm{mf}$ structural gene from the promoter. Restriction sites relevant to Southern blot are marked. (b) Left panel: Southern hybridization of Ndel-cut genomic DNA from $\mathrm{H} 293$ and $\mathrm{H} 363$, probed with $346 \mathrm{bp} \mathrm{mf}$, showing insertional mutation of the $m f$ gene, corresponding to a $7 \mathrm{~kb}$ increase in restriction fragment size in H363 ( $2 \times$ plasmid size). Right sectioned and stained with haematoxylin and eosin prior to histopathological comparison. Portions $(100 \mu \mathrm{l})$ of $10 \%$ diluted blood were plated directly onto blood agar for quantification of bacteraemia. Stability of mutation was confirmed by plating each $S$. pyogenes colony derived from H363-infected murine blood cultures onto both kanamycin and DNase agar. Furthermore, S. pyogenes colonies subcultured from H363-infected thigh muscle were replica-plated onto kanamycin blood agar plates. Sera from infected mice were frozen at $-20{ }^{\circ} \mathrm{C}$ prior to measurement of interleukin6 (IL-6) levels by ELISA (R \& D Systems).

Statistics. For in vitro experiments, the promitogenic effects of supernatants from parent strain H293 and MF-negative mutant H363 were compared by Mann-Whitney U test at each dilution tested. For in vivo studies, survival was recorded on Kaplan-Meier plots and groups were compared using the log rank test. IL-6 levels were compared using the KruskallWallis and Mann-Whitney $U$ tests. Values of $P<0.05$ were considered significant.

\section{RESULTS}

\section{Transformations}

A single kanamycin-resistant transformant was obtained following electroporation of $\mathrm{H} 293$ with plasmid DNA; this was designated H363. H293 genomic DNA yielded an 800 bp product when subjected to PCR using primers MFp and MF2, whereas this product was absent when H363 genomic DNA was used as target. In contrast, genomic DNA from both strains yielded $450 \mathrm{bp}$ and 350 bp products when primer pairs MF1/MF2 and MF1/MF2a, respectively, were used, as expected (not shown). These results suggested insertional disruption between the promoter region of $m f$ and the structural $m f$ gene. Southern hybridization following NdeI digestion of $\mathrm{H} 293$ genomic DNA using the $346 \mathrm{bp} m f$ probe showed that the wild-type $m f$ gene resided on a $1.1 \mathrm{~kb}$ restriction fragment, as predicted from the published sequence. NdeI was used as it does not cut within the $m f$ gene or the plasmid pUCMUT-mf. The size of the $m f$ hybridizing fragment increased to $8.1 \mathrm{~kb}$ in $\mathrm{H} 363$, showing that $7 \mathrm{~kb}$ of DNA (exactly twice the size of the plasmid) had inserted into this fragment (Fig. 1a, b). Tandem insertion of the plasmid was confirmed by Southern hybridization using HindIII- and SspI-digested genomic DNA from $\mathrm{H} 293$ and $\mathrm{H} 363$, followed by probing with digoxigenin-labelled $m f$ and $a p h A-3$ (Fig. $1 b)$.

\section{Growth analysis of H293 and H363}

There were no detectable differences in colony size or morphology when H293 or H363 were cultured on blood agar, nor were there any differences in beta haemolysis when the two strains were cultured either aerobically or anaerobically (not shown). Both strains

panels: HindIII-cut and Sspl-cut genomic DNA from $\mathrm{H} 293$ and H363 probed with 346 bp $m f$ (upper panel) or aphA-3 (lower panel), confirming tandem insertion of pUCMUT-mf at intended site. 


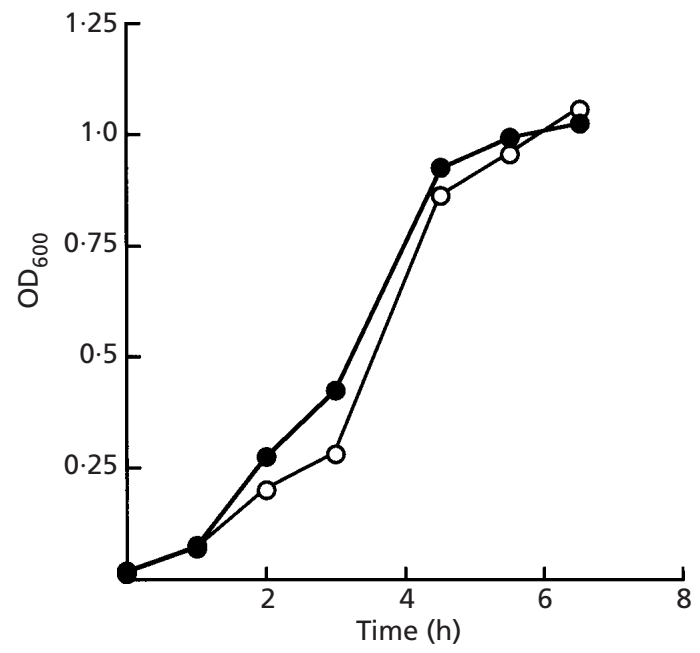

Fig. 2. Growth of wild-type S. pyogenes H293 (O) compared with mutant $\mathrm{H} 363(\mathrm{O})$ in TH broth, measured as optical density at $600 \mathrm{~nm}$.

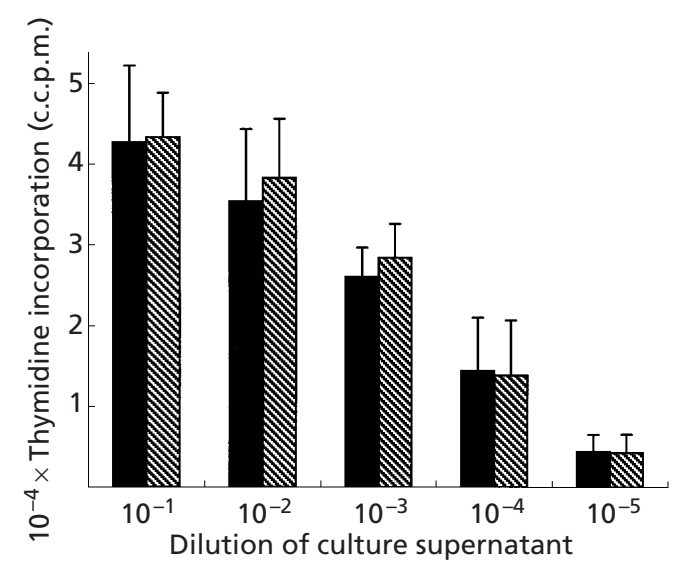

Fig. 3. Promitogenic activity of increasing dilutions of RPMI supernatants from wild-type S. pyogenes H293 (black bars) or mutant H363 (hatched bars) co-incubated with human PBMCs for $96 \mathrm{~h}$, measured by thymidine incorporation (c.c.p.m.; corrected counts per min). Each bar represents the mean of proliferation of PBMCs from three individual volunteers \pm SD. Six separate cell incubations were performed for each individual at each dilution. No statistical differences between H293 and H363 were found either for individuals or the group (Mann-Whitney $U$ test). Similar results were obtained after $72 \mathrm{~h}$ incubation in separate experiments with PBMCs from different donors.

grew comparably in THY broth; viable cell counting confirmed the results obtained by measuring optical density (Fig. 2).

\section{Promitogenic activity of $\mathrm{H} 293$ and H363}

Growth of streptococcal strains in RPMI tissue culture medium overnight did not differ between parent strain H293 $\left(7 \cdot 6 \times 10^{9}\right.$ c.f.u. $\left.\mathrm{ml}^{-1}\right)$ and H363 $\left(4.3 \times 10^{9}\right.$
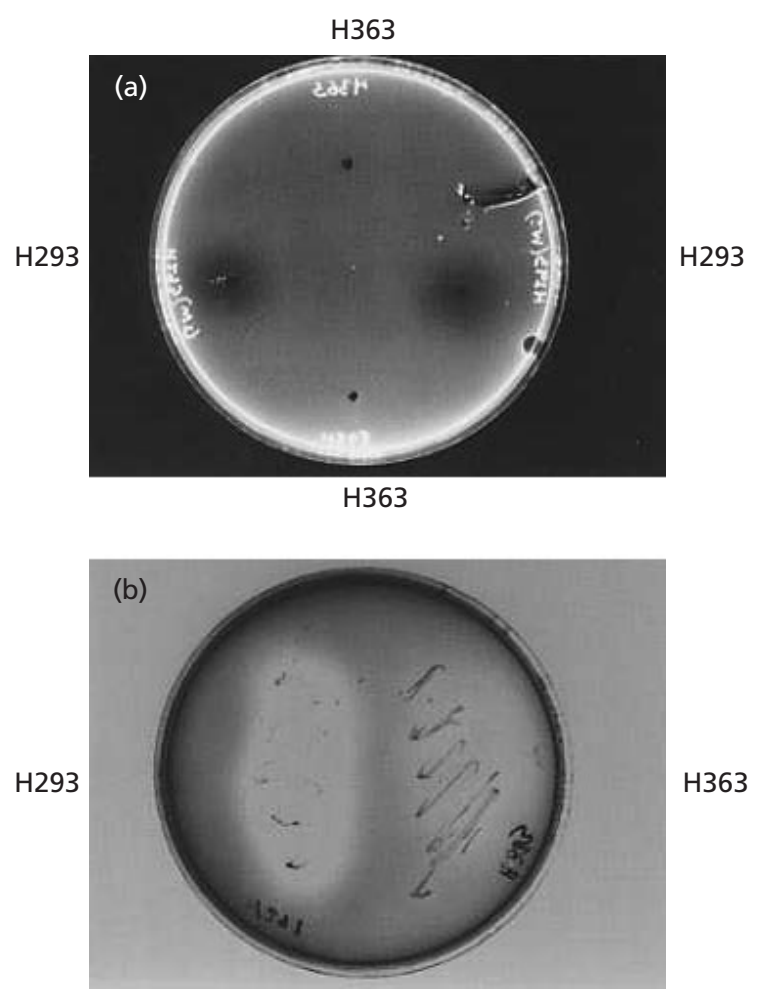

Fig. 4. DNase activity of wild-type $S$. pyogenes $\mathrm{H} 293$ compared with mutant $\mathrm{H} 363$ on methyl green conjugated DNase agar. (a) DNA hydrolysis by supernatant from $\mathrm{H} 293$ but not $\mathrm{H} 363$ incubated at $37^{\circ} \mathrm{C}$ for $5 \mathrm{~h}$. (b) $\mathrm{H} 293$ and $\mathrm{H} 363$ cultured overnight at $37^{\circ} \mathrm{C}$, photographed on a lightbox. A clear zone of DNA hydrolysis is visible around the parent strain, but not the mutant. Results shown are representative of five separate tests.

c.f.u. $\mathrm{ml}^{-1}$ ). The promitogenic activity of RPMI supernatants from $\mathrm{H} 293$ was not reduced following disruption of the $m f$ gene in $\mathrm{H} 363$, even when supernatants were diluted $10^{5}$-fold (Fig. 3). Similar results were obtained in separate experiments where supernatants were co-incubated with PBMCs from different donors for $72 \mathrm{~h}$ (not shown).

\section{DNase activity of $\mathrm{H} 293$ and $\mathrm{H} 363$}

Cell-free supernatant from and culture of H293 caused a clear zone of DNA hydrolysis on methyl green DNase agar plates. In contrast, both supernatant from and culture of H363 failed to cause any detectable DNA hydrolysis (Fig. 4). Separate experiments failed to detect any DNase activity in supernatants from $\mathrm{H} 363$ at four different $\mathrm{pH}$ values $(6 \cdot 5-8 \cdot 0)$, despite the fact that DNase activity was maintained in $\mathrm{H} 293$ even when diluted 50fold. Quantitative estimation of DNase activity using the DNase enzyme reagent (Wampole Laboratories) in a colorimetric assay also failed to detect DNase activity in H363, despite maximal DNase activity in supernatant from H293 (not shown). 
(a)

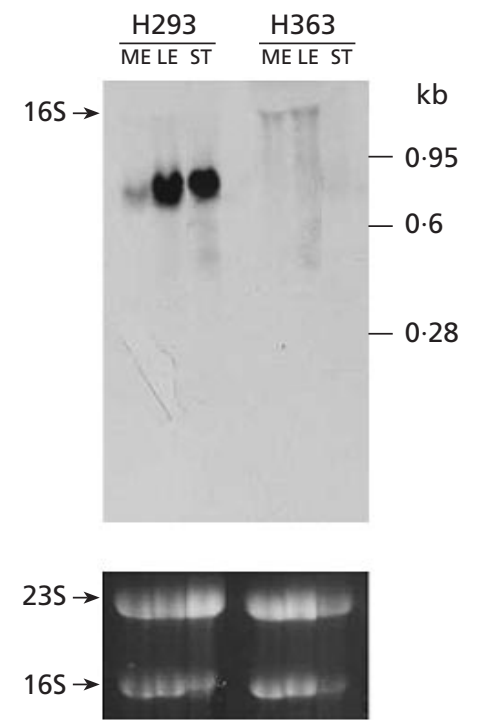

(b)

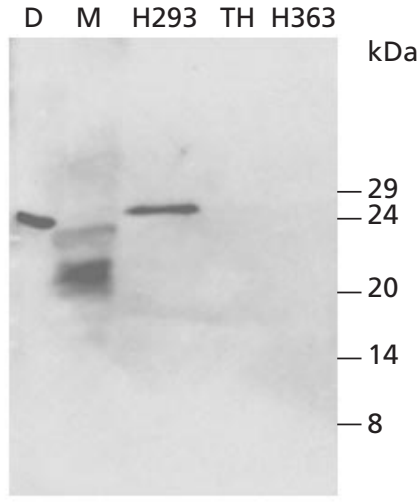

Fig. 5. (a) Upper panel: Northern analysis of RNA from wild-type S. pyogenes H293 and mutant $\mathrm{H} 363$ probed with $350 \mathrm{bp}$ digoxigenin-labelled $\mathrm{mf}$ probe. Samples were obtained from three growth phases, mid-exponential (ME), late-exponential (LE) and stationary (ST). Molecular mass markers are shown on the right. Lower panel: ethidium-bromide-stained agarose gel prior to transfer of H293 and H363 RNA to nylon. (b) Western analysis of $10 \mu \mathrm{l}$ stationaryphase supernatants from H293 compared with those from $\mathrm{H} 363$, immunoprobed with anti-DNase $B$ anti-serum. TH, TH medium alone; $\mathrm{M}$, low-molecular-mass markers; $\mathrm{D}$, $5 \mu \mathrm{l}$ commercial streptococcal DNase B. Bands corresponding to DNase B are seen in lanes D and H293 only.

\section{Northern analysis of H293 and H363}

Full length $m f$ transcripts were seen in RNA samples from late-exponential and stationary-phase broth cultures of H293, but not in those of H363 (Fig. 5a). There was no evidence of a truncated mRNA transcript in RNA from mutant H363 (corresponding to the 5' $350 \mathrm{bp}$ sequence of $m f$ remaining on the disruption vector pUCMUT-mf).

\section{Western analysis of $\mathrm{H} 293$ and $\mathrm{H} 363$ supernatants}

Anti-DNase B antibody reacted with commercially available DNase B enzyme reagent, yielding a band of the expected molecular mass. Bands identical to these were detected in supernatants from stationary-phase cultures of H293, but not in those of H363 (Fig. 5b). As expected from the Northern analysis data, there was no evidence of a $12 \mathrm{kDa}$ truncated protein product in broth from H363 (corresponding to the $5^{\prime} 350 \mathrm{bp}$ sequence of $m f$ remaining on the disruption vector pUCMUT-mf), even when broths were concentrated fivefold (not shown).

\section{In vivo infection experiments}

Survival. In two separate experiments, survival of mice following $S$. pyogenes intramuscular infection was unaffected by disruption of the $m f$ gene (Fig. 6). Infection with both $\mathrm{H} 293$ and H363 was characterized by marked swelling of the affected limb, with underlying suppuration in the soft tissues. In the first experiment, 5/15 mice infected with $\mathrm{H} 293$ demonstrated skin loss with histological evidence of necrotizing cellulitis over the infected muscle; this was not seen in H363-infected mice. Skin loss was not seen in either group when the experiment was repeated.

Bacteriology. Blood cultures were drawn from five mice in each group $48 \mathrm{~h}$ after onset of infection. 1/5 H293infected mice was bacteraemic, whereas $3 / 5$ H363infected mice were bacteraemic (range 100-200 c.f.u. ml blood $^{-1}$ ). Thus there was no detectable diminution of systemic bacterial spread following disruption of the $m f$ gene. All isolates were confirmed as group A streptococci. Blood isolates from H363-infected mice retained a kanamycin-resistant, DNase-negative phenotype when subcultured on appropriate agar. The frequency and level of bacteraemia measured $48 \mathrm{~h}$ after infection with either H293 or H363 was low compared with previous murine infections using different $S$. pyogenes strains (20). Purulent infected thigh muscle tissue obtained from surviving mice (four per group) on day 7 of infection was subcultured and shown to contain viable group A streptococci in pure culture. One hundred out of one hundred single colonies from four H363-infected mice demonstrated stable mutant phenotypes when subcultured onto kanamycin and were DNase-negative.

IL-6 levels. There were no significant differences in serum IL-6 levels between wild-type H293- and MF-negative mutant H363-infected mice ( $n=5$ mice per group). Mice infected with H293 had higher serum IL-6 levels (mean 1051 $\pm 369 \mathrm{pg} \mathrm{ml}^{-1}$ ) compared with control mice injected with saline alone (mean $119 \pm 167 \mathrm{pg} \mathrm{ml}^{-1}, P=$ 0.008). Mice infected with H363 had serum IL-6 levels of $731 \pm 280 \mathrm{pg} \mathrm{ml}^{-1}$; this was also significantly higher than saline controls.

Histopathology. Thigh muscle: a dense rim of neutrophils demarcated areas of central necrosis (including dead muscle cells and dead neutrophils) from healthy tissue in $5 / 5$ mice from each group; no differences were observed between the two groups. Liver and spleen : no significant abnormalities were noted and no differences were observed between the two groups. Kidney: extensive tubular necrosis was observed in both groups of mice, associated with sloughing of epithelial cells into the 


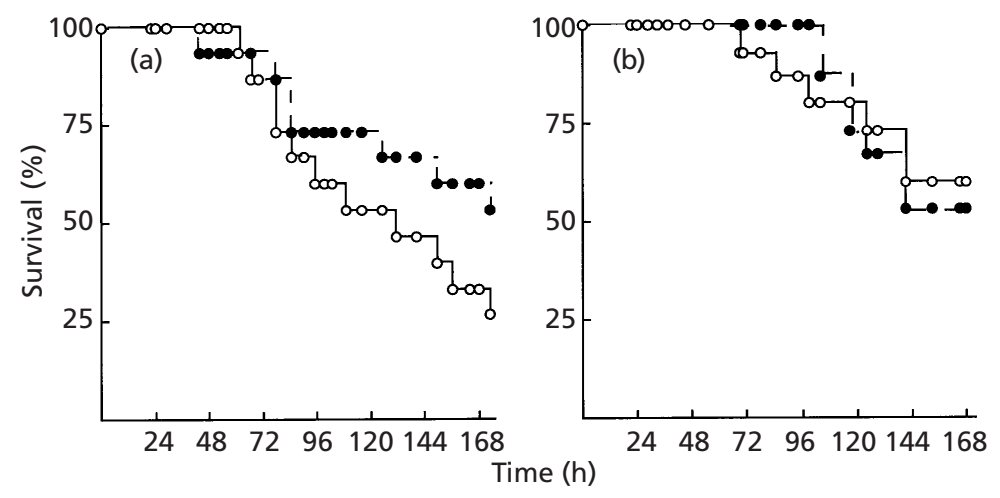

Fig. 6. Kaplan-Meier survival plots comparing survival of groups of 15 mice infected intramuscularly with either wild-type $S$. pyogenes $\mathrm{H} 293$ (O) or mutant H363 (0). Results of two separate experiments are shown. (a) Actual doses: H293-infected mice, $6.1 \times 10^{8}$ c.f.u. per mouse; H363-infected mice, $5.4 \times 10^{8}$ c.f.u. per mouse. $0.2>P>$ 0.1 , log rank test. (b) Actual doses: H293infected mice, $2.6 \times 10^{8}$ c.f.u. per mouse; H363- infected mice, $2.8 \times 10^{8}$ c.f.u. per mouse. $P>0 \cdot 2$, log rank test.

tubular lumen (casts), though glomeruli were preserved; these abnormalities were similar in extent in both groups.

\section{DISCUSSION}

Disruption of the $m f$ gene in this $S$. pyogenes strain has demonstrated that the exotoxin MF, also known as SPEF, does not contribute significantly to the superantigenic activity of $S$. pyogenes when compared with other superantigens encoded by this strain, namely SMEZ and SPEG. Furthermore, we have shown that disruption of the gene encoding MF resulted in loss of DNase activity, associated with loss of DNase B production, confirming previous reports that the two proteins are the same. Finally, we have examined the importance of $\mathrm{MF} / \mathrm{DNase}$ as a virulence factor in invasive streptococcal infection and have demonstrated that, at least in this strain, MF/DNase alone is not a critical virulence factor.

MF has been studied largely because of its reported superantigenic qualities. However, amongst the array of constitutive superantigens expressed by $S$. pyogenes strain H293, the mitogenic activity of MF was undetectable in the streptococcal supernatants used in our studies. Previously, we disrupted the gene encoding SPEA in S. pyogenes strain H305 and found a small but consistent diminution in promitogenic activity of the SPEA-negative mutant (Sriskandan et al., 1999). In contrast, in this work, disruption of MF did not lead to any diminution of promitogenic activity. We considered the possibility that the parent strain $\mathrm{H} 293$ may not produce MF. However, separate transcription studies confirmed that $m f$ mRNA was produced by $\mathrm{H} 293$ at a level similar to that seen in other strains (not shown). Furthermore, supernatants used in these studies were from stationary-phase cultures, a growth phase when $m f$ transcription is known to be maximal (Unnikrishnan et al., 1999) and proliferation studies were carried out at a time point when mitogenic effects of MF are thought to be maximal. We considered the possibility that the mutant H363 could produce a truncated MF product from the $350 \mathrm{bp}$ sequence encoded by the disruption vector; this was discounted because no such product was detected at either RNA or protein levels. NorrbyTeglund et al. (1994b) showed that T cell proliferation induced by purified MF was HLA class II-dependent and did not require conventional antigen processing, consistent with the definition of a superantigen. Subsequent proliferation studies using recombinant toxins have confirmed that recombinant $(\mathrm{r}) \mathrm{MF}$ is mitogenic and can cause $\mathrm{T}$ cell proliferation compared with $\mathrm{rSPEB}$, the streptococcal cysteine protease, in a class II-dependent TCR V $\beta$-restricted manner, similar to superantigens (Toyosaki et al., 1996). However, in the same experiments $10^{5}$-fold more rMF was required to achieve levels of proliferation which were comparable with the recognized superantigens rSPEA or rSPEC. Although care must be taken when interpreting data from toxins expressed recombinantly, the data do suggest that MF lacks the potent T-cell-stimulating properties attributed to some of the other bacterial superantigens. Two studies have, however, shown that MF or rMF can induce significant cytokine production when co-incubated with human PBMCs, thus this secreted protein may yet contribute to the proinflammatory properties of S. pyogenes (Norrby-Teglund et al., 1994c; Toyosaki et al., 1996).

It has long been suspected that the genes encoding MF and DNase B are identical. A GenBank BLAST search using the sequence for MF published in 1993 (GenBank accession no. D13428) failed to locate registered sequences with significant homology. However, sequences listed under 1996 patent codes and annotated as streptococcal DNase B (GenBank accession no. A49208) demonstrate identity to $m f$ when aligned. We considered the possibility that two virtually identical genes might co-exist in the $S$. pyogenes genome, though this was excluded in the wild-type strain we studied as only a single $1 \mathrm{~kb}$ fragment hybridized to the $m f$ probe following NdeI genomic digestion. Identity at the protein level was first suggested by Iwasaki et al. (1997), who demonstrated that recombinant MF had DNase activity in addition to the mitogenic actions shown in earlier work. A subsequent study from Sweden showed that the two proteins were immunologically identical using polyclonal antisera (Eriksson et al., 1999). Our study provides clear confirmatory evidence that a single structural gene within the streptococcal genome encodes the proteins MF and DNase B. Furthermore, both $m f$ and DNase B sequences map to a single location in the now completed Oklahoma S. pyogenes M1 genome 
database, confirming the identity of these genes in the this strain also (Suvorov \& Ferretti, 1996).

We were initially surprised to find that DNase production was undetectable in the mutant strain $\mathrm{H} 363$, and considered the possibility that the insertional mutation induced in this study had resulted in a polar effect on other DNase-regulating genes adjacent to the target gene, or that the target gene formed part of a larger DNase-regulating operon. However, transcription studies have repeatedly shown that the transcript produced by the $m f$ gene is monocistronic in this and other $S$. pyogenes strains and a polar effect or operon appears unlikely (Unnikrishnan et al., 1999). We therefore conclude that, in the strain studied, DNase B is the only detectable DNase produced. S. pyogenes can produce four serologically distinct DNases, A-D, which, with the exception of DNase D (Podbielski et al., 1996), have not been studied at the molecular level.

It is suggested that streptococcal DNase activity may facilitate liquefaction of pus and spread through tissue planes during invasive streptococcal infection (Bisno, 1995). Because of the evidence that MF might be involved in vascular permeabilization in sepsis and seroepidemiological data which implicate MF as a virulence factor (Matsumoto et al., 1999; Norrby-Teglund et al., 1994a), we elected to compare the wild-type and isogenic MF/DNase-negative mutant strains in a mouse model of invasive necrotizing fasciitis. Disruption of $m f$ did not, however, affect virulence of $S$. pyogenes with regard to host survival, histopathological change or bacterial growth during invasive infection and we have shown unequivocally that the induced mutation in strain H363 is rigorously stable in vivo. Podbielski et al. (1996) previously disrupted the DNase D gene in an M49 S. pyogenes strain and found opsonophagocytosis resistance unaltered, even though DNase activity was totally abrogated; in vivo studies of virulence were not reported. To our knowledge, this is the first study to address the specific role of a Gram-positive DNase in a sepsis model, though Staphylococcus aureus mutants deficient in a range of exoproteins including DNase are known to exhibit attenuated virulence (Bogni et al., 1998). It is possible that $\mathrm{DNase} B$ has a more prominent role in different forms of infection or in throat colonization, or, indeed, in different streptococcal strains. The parent strain, H293, used in the current study induced a pattern of pyogenic change in murine thigh muscle plus severe tubular renal damage, which contrasts with more invasive changes seen previously with other S. pyogenes strains (Sriskandan et al., 1996). It is noteworthy that mutation of the gene encoding MF was not achieved in an M1T1 S. pyogenes strain despite demonstrable bacterial cell competency (authors' unpublished observations); we cannot exclude the possibility that, in some strains, DNase activity might be essential. The development of a stable isogenic mutant lacking detectable DNase activity should facilitate studies to analyse the function of this group of enzymes. Furthermore, to avoid future confusion, a standardized nomenclature for this protein should be agreed upon.

\section{ACKNOWLEDGEMENTS}

This work was funded by a Medical Research Council (UK) Clinician Scientist award to S.S. (grant no. G108/245).

We are grateful to Dr Dieter Gerlach for providing purified protein and anti-DNase B serum, to Peter Fenwick for technical assistance in ELISA, and also to the University of Oklahoma Streptococcus pyogenes genome sequencing project for making the M1 sequence available on the Internet.

\section{REFERENCES}

Bisno, A. L. (1995). Streptococcus pyogenes. In Principles and Practice of Infectious Diseases, pp. 1786-1799. Edited by G. L. Mandell, J. E. Bennett \& R. Dolin. New York: Churchill Livingstone.

Bogni, C., Segura, M., Giraudo, J., Giraudo, A., Calzolari, A. \& Nagel, R. (1998). Avirulence and immunogenicity in mice of a bovine mastitis Staphylococcus aureus mutant. Can J Vet Res 62, 293-298.

Caparon, M. G. \& Scott, J. R. (1991). Genetic manipulation of pathogenic streptococci. Methods Enzymol 204, 556-586.

Davies, H. D., McGeer, A., Schwartz, B., Green, K., Cann, D., Simor, A. E. \& Low, D. E. (1996). Invasive group A streptococcal infections in Ontario, Canada. Ontario group A streptococcal study group. N Engl J Med 335, 547-554.

Eriksson, A., Eriksson, B., Holm, S. E. \& Norgren, M. (1999). Streptococcal DNase B is immunologically identical to superantigen SpeF but involves separate domains. Clin Diagn Lab Immunol 6, 133-136.

Gerber, M. A., Kaplan, E. L., Gastanaduy, A. S., McKay, C. \& Wannamaker, L. W. (1980). The immunologic response to group A streptococcal upper respiratory tract infections in very young children. J Pediatr 96, 374-379.

Herman, A., Kappler, J. W., Marrack, P. \& Pullen, A. M. (1991). Superantigens: mechanism of T-cell stimulation and role in immune responses. Annu Rev Immunol 9, 745-772.

Iwasaki, M., Igarashi, H., Hinuma, Y. \& Yutsudo, T. (1993). Cloning, characterization and overexpression of a Streptococcus pyogenes gene encoding a new type of mitogenic factor. FEBS Lett 331, 187-192.

Iwasaki, M., Igarashi, H. \& Yutsudo, T. (1997). Mitogenic factor secreted by Streptococcus pyogenes is a heat-stable nuclease requiring His ${ }^{122}$ for activity. Microbiology 143, 2449-2455.

Matsumoto, M., Ishikawa, N., Saito, M., Shibayama, K., Horii, T., Sato, K. \& Ohta, M. (1999). Streptococcal pyrogenic exotoxin F (SpeF) causes permeabilization of lung blood vessels. Infect Immun 67, 4307-4311.

Norrby-Teglund, A., Pauksens, K., Holm, S. E. \& Norgren, M. (1994a). Relation between low capacity of human sera to inhibit streptococcal mitogens and serious manifestations of disease. $J$ Infect Dis 170, 585-591.

Norrby-Teglund, A., Newton, D., Kotb, M., Holm, S. E. \& Norgren, M. (1994b). Superantigenic properties of the group A streptococcal exotoxin SpeF (MF). Infect Immun 62, 5227-5233.

Norrby-Teglund, A., Norgren, M., Holm, S. E., Andersson, U. \& Andersson, J. (1994c). Similar cytokine induction profiles of a novel streptococcal exotoxin, MF, and pyrogenic exotoxins A and B. Infect Immun 62, 3731-3738.

Podbielski, A., Zarges, I., Flosdorff, A. \& Weber-Heynemann, J. (1996). Molecular characterization of a major serotype M49 group A streptococcal DNase gene (sdaD). Infect Immun 64, 5349-5356. 
Proft, T., Moffatt, S. L., Berkahn, C. J. \& Fraser, J. D. (1999). Identification and characterization of novel superantigens from Streptococcus pyogenes. J Exp Med 189, 89-101.

Sriskandan, S., Moyes, D., Buttery, L. K., Krausz, T., Evans, T. J., Polak, J. \& Cohen, J. (1996). Streptococcal pyrogenic exotoxin A release, distribution, and role in a murine model of fasciitis and multiorgan failure due to Streptococcus pyogenes. J Infect Dis 173, 1399-1407.

Sriskandan, S., Unnikrishnan, M., Krausz, T. \& Cohen, J. (1999). Molecular analysis of the role of streptococcal pyrogenic Exotoxin A (SPEA) in invasive soft-tissue infection resulting from Streptococcus pyogenes. Mol Microbiol 33, 778-790.

Suvorov, A. N. \& Ferretti, J. J. (1996). Physical and genetic map of an M1 type 1 strain of Streptococcus pyogenes. J Bacteriol 178, $5546-5549$.
Toyosaki, T., Yoshioka, T., Tsuruta, Y., Yutsudo, T., Iwasaki, M. \& Suzuki, R. (1996). Definition of the mitogenic factor (MF) as a novel streptococcal superantigen that is different from streptococcal pyrogenic exotoxins A, B, and C. Eur J Immunol 26, 2693-2701.

Unnikrishnan, M., Cohen, J. \& Sriskandan, S. (1999). Growth phase-dependent expression of virulence factors in an M1T1 clinical isolate of Streptococcus pyogenes. Infect Immun 67, 5495-5499.

Zurawski, C. A., Bardsley, M. S., Beall, B., Elliot, J. A., Facklam, R., Schwartz, B. \& Farley, M. M. (1998). Invasive group A streptococcal disease in metropolitan Atlanta: a population-based assessment. Clin Infect Dis 27, 150-157.

Received 16 May 2000; revised 28 July 2000; accepted 3 August 2000. 